\title{
Designing for Positive Emotional Responses in Users of Interactive Digital Technologies: A Systematic Literature Review
}

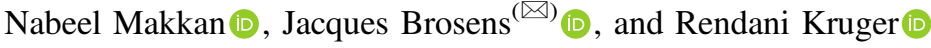 \\ Department of Informatics, University of Pretoria, Pretoria, South Africa \\ \{nabeel.makkan, jacques. brosens, \\ rendani.kurger\}@up.ac.za
}

\begin{abstract}
In this study, 20 papers were reviewed to identify means, methods, techniques, tools or other interventions that practitioners may use to positively influence the affective state of users of digital interactive technologies. A systematic literature review was conducted in order to find such interventions. A literature background covering concepts from the fields of Human-computer interaction, as well as certain concepts from the field of psychology that is relevant to this study is provided. Search criteria were determined and used to identify research papers from various academic resources. Four categories of intervention categories were identified from the papers reviewed, which can be applied to interactive digital technologies by practitioners in order to evoke positive affect on users. These four categories were aesthetics, affective computing, need fulfilment and novel interaction techniques. The identified categories for practitioners were consolidated alongside metadata such as the types of publications of the reviewed papers, the regions the studies were conducted and the growth in the number of studies.
\end{abstract}

Keywords: User experience · Human-Computer Interaction · Positive affect • Hedonic quality $\cdot$ Delight $\cdot$ Systematic literature review

\section{Introduction}

Functionality and usability have long been the main considerations taken in to account by practitioners developing interactive digital technologies [1]. However, user experience (UX) design has moved towards a more experiential approach, and no longer focuses on just functionality and usability. Understanding the user that interacts with technology [2] has led to more attention being paid by practitioners to psychological aspects that influence a user's perception of an experience.

There are two attributes of UX that influence the appeal, pleasure and satisfaction in a user, pragmatic attributes, i.e. the attributes that fulfil a user's functional and usability needs, and hedonic attributes, i.e. those attributes that fulfil a user's psychological needs related to emotion and pleasure [3]. Despite both hedonic and pragmatic qualities having a relation to positive affect, hedonic quality appears to have a stronger relationship to positive affect than the pragmatic quality of a user experience [4]. 
Given the effect of positive affect and hedonic qualities on the appeal of a technology to a user, the following research question can be raised: "What practices, techniques or interventions can a practitioner implement to evoke positive affective responses from users of a digital interactive technology?". The aim of this review is to evaluate existing research in the discipline of human-computer interaction (HCI) and user experience to identify the different interventions that have been put forward by researchers specifically intended to improve the emotional responses evoked in users of digital interactive technologies. The focus of this review will not be on the functionality or usability (pragmatic quality) aspects, but instead on the pleasurable and emotional aspects (hedonic quality) of user experiences.

This review firstly describes some of the background knowledge on HumanComputer Interaction, Human-Centered Design, User Experience and the Hedonic/Pragmatic model of UX. The literature background also investigates some concepts from psychology, such as the pleasure-arousal-dominance model [5, 6], and the ten psychological needs [7]. The selection process of articles used for the review is described in Sect. 3 including search terms, sources, as well as the data extraction process. The extracted research was then reviewed to answer the research question.

\section{Literature Background}

This section describes key concepts that are relevant in this research, such as HumanComputer Interaction (HCI), Human-centered design (HCD), User Experience (UX) and The Hedonic/Pragmatic model. While the focus of this study is in the context of HCI and UX, answering the research question involved understanding certain concepts or models that relate to a user's emotions from a psychology perspective, and, therefore, this section also describes relevant concepts from the field of psychology.

HCI is a broad term for the study of the interaction between humans and computers [8]. Two key considerations in designing human computer interfaces are functionality and usability [8]. The functionality of a technology is defined by what the technology can do, either actions or services, for the user to achieve goals, while the usability of the system is the degree to which the user can efficiently use a particular function or set of functions provided by the system [9]. The functionality of a technology is only fully realised if it is usable by the intended user of the technology [10], this is an important consideration in the development of systems.

HCD is defined as "an approach to interactive systems development that aims to make systems usable and useful by focusing on the users, their needs and requirements, and by applying human factors/ergonomics, and usability knowledge and techniques" [11]. HCD, originates from User-Centered Design (UCD), is a concept coined by Norman in 1986, and later built on in his book "The Psychology of Everyday Things" [12].

In UCD the user is at the center of the design process [12]. HCD aims to improve the design of technologies/systems by understanding the needs, emotions, abilities and experiences of the intended users [13]. Developing a system that meets the needs of the user through HCD effectively can evoke positive experience from users. 
UX is defined as the evaluative feeling experienced by an end user during their interaction with a digital technology [14]. While traditional HCI studies focus more on the usability or utilitarian aspects of user's interaction with a digital technology, UX studies include other, non-utilitarian aspects of these interactions, such as a users' emotions, the meaning and value of the interactions to the user [15].

The hedonic/pragmatic model is a model of UX that recognizes that people perceive their experience of interactive products in two dimensions, pragmatics and hedonics [16]. Pragmatics refers to the product's attributes which provide utility to the user, such as completing a specific task that fulfils a functional need of the user [17]. Hedonics are the attributes of a product that fulfil the psychological needs of the user, those needs of enjoyment or pleasure, self-expression and even provocation of memories [16].

A model to study hedonics is the Pleasure-Arousal-Dominance (PAD) model by Mehrabian and Russell [5] which suggest that there are three dimensions that represent the affective response of a user to a stimulus: pleasure which is the scale of the pleasantness of the affective response to a stimulus, arousal which represents an individual's degree of excitement, and dominance which is the extent individuals feel as if they are in control $[5,6]$. The PAD model has been applied in numerous studies in the field of HCI to measure user intention, behavior and response in various contexts like online gaming [18], online marketplaces [19] and even in UX [20]. Some other models in psychology like the 10 psychological needs by Sheldon, Elliot, Kim and Kasser [7] have also been used in UX to understand human needs that influence their experiences.

In summary, when systems are designed and developed to provide specific functionality to end users, the usability and the experience of those end users should also be considered [10]. Approaches such as HCD involve users throughout the design process, in order to take in to consideration their needs, abilities and emotions [13]. UX design that considers the emotional wellbeing of the user can make a technology more appealing, easier to use and will ultimately improve the effectiveness and usefulness of the end product $[2,4]$.

\section{Research Method}

This study followed a systematic literature review (SLR) process [21]. The research question, derived from the title was: "What practices, techniques or interventions can a practitioner implement to evoke positive affective responses from users of a digital interactive technology?".

The IEEE Xplore, Ebscohost, SpringerLink, ACM Digital Library, ScienceDirect, ResearchGate, Google Scholar, Web of Science, Scopus and Semantic Scholar academic databases were used in the SLR process.

The search term used for the SLR to search academic research databases was: ("user experience" OR "user (NEAR/2) experience" OR UX) AND (tool* OR Technique* OR Method* OR Practic* OR Intervention OR Mean*) AND (emotion* OR hedoni* OR Pleasur* OR Delight* OR Enjoy* OR arous* OR Domin* OR "positive affect" $\mathbf{O R}$ affect*). 
Once academic sources were extracted from the databases using the search criteria, the sources were filtered based on selection criteria. Sources were included when:

- They were published in English between 1989 and 2019.

- They were peer review academic articles related to HCI and or UX.

- They reported on the effectiveness of a specific practice, technique or method that can be implemented in a digital technology to invoke positive affect or improve hedonic quality.

Articles were excluded from the study if they did not meet the inclusion criteria or if they were duplicates. The SLR process involved (1) using the search terms in each academic database to obtain a preliminary set of articles (2) the removal of duplicate articles (3) the exclusion of articles based on the selection criteria (4) screening and filtering of articles through abstract reviews to determine relevance. Initially 323 articles were identified, 301 were excluded and 20 formed part of the final set of articles used in this study [21].

\section{Results}

This section contains the findings of this review. The results were analyzed for information such as the types of articles reviewed, the journals the articles were published in, and the geographical spread of the research reviewed. The contents of each article were then analyzed through a thematic analysis to identify the themes and to categorize each article.

\subsection{Sources Overview}

20 articles were included for review in this study, 16 of the 20 articles were peerreviewed journal articles, and four were conference proceedings. Four papers $(20 \%)$ included were published in the International Journal for Human-Computer Interaction, three $(15 \%)$ in the International Journal of Human-Computer Studies, the rest of the papers were extracted from other journals (1 paper from each individual journal).

Geographically, seven studies included were conducted in Asia, three in China, two in Singapore, one in Japan, and one in South Korea. Seven studies were based in the North American region, five in the USA and two in Canada. Six studies were conducted in Europe, three studies in Germany, and one study each in Finland, France and Italy. There were no studies that included from Africa, South America or the Middle East. There has been an increase in the number of studies released on the subject over the last three decades. Only one of reviewed papers $(5 \%)$ were published in the period of 1990 to 1999 . Four of the papers reviewed (20\%) were published in the period of 2000-2010, and 15 papers (75\%) were published between 2010 and 2019.

\subsection{Findings}

The 20 articles were analyzed thematically to categorize them based on the way they suggest designing for positive emotional responses in digital technologies. The 
identified themes are the specific implementations, practices, or guidelines practitioners can implement in digital interactive technologies to evoke positive affect in users. Many of the papers focused on a specific characteristic, implementation or technique and their influence on the hedonic quality of the user experience or on positive affect invoked on users. They were then grouped together and then categorized into a theme. The identified themes were aesthetics (9 papers), affective computing (6), novel interaction techniques (1 paper) and need fulfillment ( 4 papers). These themes are depicted in Table 1.

Table 1. Themes and categories used.

\begin{tabular}{l|l|l}
\hline Theme & Categories & Articles \\
\hline Aesthetics & General aesthetics & {$[22-25]$} \\
& Animations and effects & {$[26-28]$} \\
& Color appeal & {$[29,30]$} \\
\hline Affective computing & Affective language & {$[31,32]$} \\
& Anthropomorphism & {$[33-35]$} \\
& Conversational interactions & {$[36]$} \\
\hline Novel interaction techniques & Novel interaction techniques & {$[37]$} \\
\hline Need fulfillment & General need fulfilment & {$[4,38]$} \\
& Need fulfilment through gamified systems & {$[39,40]$} \\
\hline
\end{tabular}

Aesthetics. From the 20 papers reviewed, 9 studied the influence of aesthetics on UX. These were further categorized under general aesthetics, animations and effects, color appeal.

General Aesthetics. General aesthetics involved studying the influence of aesthetics in general on the affective responses of users, rather than a specific aesthetic quality or implementation. Bhandari et al. [24] performed a study on classical aesthetics (described with the dimensions of symmetry, clarity and cleanliness) and expressive aesthetics (with the dimensions of originality, creativity and special effects), and found that both classical and expressive aesthetics had a significant effect on user emotional states. Classical aesthetics significantly affects the valence (pleasure) dimension of emotions, while expressive aesthetics reflects in the arousal dimension of emotion [24]. Another paper found that beautiful web-pages had a similar effect as pleasant pictures, and ugly web-pages to that of unpleasant pictures [23]. Ugly webpages induced a negative affective state, while pleasant web pages influenced the affective state of the user towards the positive end [23]. A study on the visual complexity of a webpage found that it bears a positive correlation to the arousal and pleasantness experienced by the user [25]. Design aesthetics of an m-commerce site was shown to positively influence the perceived enjoyment by the user, and it was shown that perceived enjoyment is as important to the loyalty of a user as perceived usefulness [22]. Based on these studies it can be said that practitioners can use different general aesthetic characteristics or interventions, such as symmetry, clarity and cleanliness, and expressive aesthetic qualities (creativity, special effects and originality) to evoke a 
positive affective state in users [24]. Practitioners that implement attractive design aesthetics are more likely to influence the perceived enjoyment of users [22, 23].

Animations and Effects. Articles classified as animations and effects studied the influence of animations and other special effects on the affective responses of users. A website containing both static and animated content is perceived as hedonically superior [28]. A study on the effectiveness of parallax scrolling found that it did not affect the perceived usability, aesthetic or satisfaction of the user experience but did have a significant effect on the perceived "fun" experienced of the participant [26]. Another study on the implementation of parallax effects in an interactive interface found that including such effects on an interface made it more likely to be perceived as "cool" or "vivid" by users [27]. Based on these studies, implementing animations, such as parallax scrolling effects, can allow practitioners to increase the likelihood of inducing positive affect in the users.

Color Appeal. Articles classified under color appeal focused on the relationship between the colors used in an interface and the affective state of users. A study on color appeal of an e-commerce site found that color had a significant link with trust and satisfaction when using an e-commerce platform [30]. The study also tested the effect of different colors on multiple users' (from different cultural backgrounds), and found that certain colors can create an adverse reaction in users [30]. Bonnardel et al. [29] studied the effect of colors on designers and users' cognitive processes. Practitioners should therefore carefully consider the combinations of color that are utilized on their interfaces.

Affective Computing. Six papers reviewed studied the impact of affective computing, which were categorized in to 3 sub-categories, namely affective language, anthropomorphism, and conversational interactions. These papers studied the influence of using emotional, more human-like language or human-like expressions on the affective responses of users.

Affective Language. Affective language refers to the use of language that appeals to the emotion of the reader/listener. The studies in this sub-category focused on the influence of this implementation on the affective responses of users. A paper on the effect of flattery from computers found that both flattery and sincere praise from the computer had a significant effect on the affective response of the user, i.e. users had greater positive affect obtained from the interaction, when compared to generic feedback from the computer [32]. Flattery and sincere praise also improved the user's perception of their own performance, as well as the satisfaction, enjoyment and evaluation of the computer's performance [32]. Another study found that receiving apologetic messages from a computer when something goes wrong made users less likely to experience frustration when compared to non-apologetic or neutral messages [31]. Based on these studies, practitioners could illicit positive affective responses from users through utilising affective language, such as apologetic messages when displaying error messages [31] and providing sincere praise to users through the interface during interactions [32].

Anthropomorphism. Anthropomorphism is described as attributing human-like characteristics or behavior to non-human entities [41]. The articles in this section studied 
the influence of implementing human-like characteristics to interfaces on the affective responses of the user. Mancini et al. [33] studied the effects of implementing a laughing virtual character in an interface and found that the presence of such character increased their level of activation and puzzlement. Qiu and Benbasat [34] studied the effects of anthropomorphic embodiments in the interfaces of product recommendation agents and found that it can increase the user's perceived enjoyment and trust in the recommendation agent. A study on anthropomorphism in digital voice assistants found that a more human-like interaction between the voice assistant and the user positively influenced the likeability of the digital voice assistant, which in turn also influenced the user's intention to use the assistant again [35]. Based on the above research, practitioners can, therefore, influence the affective responses of users through implementing virtual characters, or other anthropomorphic means/techniques or tools in their interfaces.

Conversational Interactions. A study on conversational agents found that selfdisclosure (communicating information about one's self) and reciprocity (adequate responses and continuance of conversation) with the conversational agents significantly influences the perceived enjoyment of the interaction, while interactional enjoyment also influenced the user satisfaction experienced when interacting with the conversational agent [36]. While there is only one study in this sub-category, practitioners could still implement more conversational interactions with users as the study does show that it can significantly influence affective responses.

Need Fulfilment. Four papers studied the effects of need fulfilment. These papers studied the influence of fulfilling user needs on the affective responses of users. These were categorized under general need fulfilment or need fulfilment through gamified systems.

General Need Fulfilment. These papers studied the influence of satisfying the needs as identified by Sheldon et al. [7] on the affective state of the user. Hassenzahl et al. [4] studied the effects of fulfilment of the needs identified by Sheldon and King [42] on the affect and user experience, and found that satisfaction of each need is strongly correlated to positive affect. Hassenzahl et al. [38] studied need fulfilment and positive affect further. Practitioners can evoke positive affective responses in users through fulfilling user needs (such as those identified by Sheldon and King).

Need Fulfilment Through Gamified Systems. The studies in this sub-category look at how gamification can fulfil human needs and as a result influence the affective state of the user positively. Hamari and Koivisto [39] and Suh et al. [40] studied the effects of gamification on user engagement as well as the needs fulfilled by applying gamifying interactions. Gamified systems can fulfil the user needs identified by Sheldon and King [42]. Suh et al. [40] found that gamified elements such as gaining points, levels and badges can positively influence the satisfaction of the user's need to competence and autonomy. Gamified elements can thus be utilized by practitioners to fulfil user needs. Therefore, as was shown by Hassenzahl et al. [4, 38], satisfying these needs can positively influence positive affective responses from users. 
Novel Interaction Techniques. One paper, by Dou and Sundar [37], studied the effects of implementing different interaction techniques. Adding swiping gestures to a web interface had a significant effect on the perceived enjoyment of a mobile website [37]. Based on the above study, practitioners that implement interaction techniques such as swiping and clicking can, therefore, influence the perceived enjoyment by the user. While, once again, the findings of this study were not evidenced due to the lack of further research, practitioners may still find benefit in implementing these techniques into their products.

\section{Discussion}

Aesthetics was the most popular theme covered by the articles reviewed, comprising of nine (9) of the 20 articles reviewed (36\%), followed by affective computing, comprising of six (6) of the 20 articles reviewed (30\%). Four (4) studies were on the influence of need fulfilment on the affective responses of users. Only one (1) article studied some sort of interaction technique on the affective responses of users.

Based on the above, aesthetic aspects of an interface are more likely to evoke a positive affective response within users, as there is evidence to support the influence of aesthetics qualities, techniques or implementations on the user's affective response [22-30]. Practitioners will likely find that they can evoke affective responses in users through the aesthetics of the interface of a digital technology that they are developing.

Practitioners may also implement affective computing techniques, as there is also evidence to support their influence on the affective responses of users [31-36].

Need fulfilment also influences the affective responses of users, and as such satisfying these needs of users' (such as those identified by Sheldon and King) [4, 38]. Practitioners can evoke affective responses from users through the implementation of techniques such as gamification [39, 40].

Finally, while there is only one study supporting the influence of an interaction technique on the affective responses of users, implementing those techniques may still allow practitioners to evoke positive affect in users. Further studies into interaction techniques and affective responses will benefit practitioners. It is important to note that practitioners do not need to implement every identified means of evoking affective response from users in their technologies, as certain implementations are relevant to specific types of applications. For example, the findings on conversational interaction may only be relevant to those interactive systems that involve users 'conversing' with them, such as digital voice assistants or chatbots. Practitioners should identify the ideal implementations that would best suit the interactive technology that they are developing.

While the number of studies published over the last three decades that were relevant to this study has steadily increased, there were still relatively few papers that were available to review in order to answer the research question of this paper. Certain interventions, while shown to positively influence affective responses, were studied in very few papers, their findings were not verified with other research or similar results. Further studies in this area of research should allow for new knowledge that can be utilized by practitioners of UX. 


\section{Conclusion}

This paper provided a systematic literature review on existing research available in order to identify methods, means, techniques and tools that practitioners can implement into their digital interactive technologies to evoke affective responses from users. 20 articles were obtained through various digital libraries, on which thematic analysis was conducted in order to identify themes that relate to the research question. 16 papers of the papers selected were journal articles and four papers were conference proceedings. In reviewing the selected papers, four overarching themes were identified, aesthetics, affective computing, need fulfilment and novel interaction techniques.

Furthermore, three sub-categories were identified for both the theme of aesthetics and affective computing and two were identified for the theme of need fulfilment. These papers provided studies on the influence of specific techniques, tools, means or methods on the affective responses of the users of digital interactive technologies. This study allows practitioners identify various interventions that UX practitioners can use in their digital interactive technologies to positively influence the affective responses/state of the user. Furthermore, this study identifies the relatively low quantities of studies in the focus area of this research. Further academic research in this area may allow for practitioners to identify more interventions or methods that can be implemented in their products.

\section{References}

1. Angeli, A.D., Sutcliffe, A., Hartmann, J.: Interaction, usability and aesthetics: what influences users' preferences? p. 10 (2006)

2. Zimmermann, P.G., Gomez, P., Danuser, B., Schär, S.G.: Extending usability: putting affect into the user-experience, p. 7 (2006)

3. Hassenzahl, M., Platz, A., Burmester, M., Lehner, K.: Hedonic and ergonomic quality aspects determine a software's appeal. In: Proceedings of the SIGCHI Conference on Human Factors in Computing Systems - CHI 2000, pp. 201-208. ACM Press, The Hague (2000). https://doi.org/10.1145/332040.332432

4. Hassenzahl, M., Diefenbach, S., Göritz, A.: Needs, affect, and interactive products - facets of user experience. Interact. Comput. 22, 353-362 (2010). https://doi.org/10.1016/j.intcom. 2010.04.002

5. Mehrabian, A., Russell, J.A.: An Approach to Environmental Psychology. M.I.T. Press, Cambridge (1974)

6. Russell, J.A., Mehrabian, A.: Evidence for a three-factor theory of emotions. J. Res. Pers. 11, 273-294 (1977). https://doi.org/10.1016/0092-6566(77)90037-X

7. Sheldon, K.M., Elliot, A.J., Kim, Y., Kasser, T.: What is satisfying about satisfying events? Testing 10 candidate psychological needs. J. Pers. Soc. Psychol. 80, 325-339 (2001)

8. Zhang, P., Carey, J., Te'eni, D.: Human-Computer Interaction: Developing Effective Organizational Information Systems. Wiley, Hoboken (2007)

9. Karray, F., Alemzadeh, M., Abou Saleh, J., Nours Arab, M.: Human-computer interaction: overview on state of the art. Int. J. Smart Sens. Intell. Syst. 1, 137-159 (2008). https://doi. org/10.21307/ijssis-2017-283 
10. Shneiderman, B., Plaisant, C.: Designing the User Interface: Strategies for Effective HumanComputer Interaction, 5th edn. Pearson, New Delhi (2016)

11. International Organization for Standardization: ISO 9241-210:2010 Ergonomics of humansystem interaction - Part 210: Human-centred design for interactive systems. International Organization for Standardization (2010)

12. Norman, D.A.: The Design of Everyday Things. Basic Books, New York (1988)

13. Giacomin, J.: What is human centred design? Des. J. 17, 606-623 (2014). https://doi.org/10. 2752/175630614X14056185480186

14. Hassenzahl, M.: User experience (UX): towards an experiential perspective on product quality. In: Proceedings of the 20th International Conference of the Association Francophone d'Interaction Homme-Machine on - IHM 2008, p. 11. ACM Press, Metz (2008). https://doi.org/10.1145/1512714.1512717

15. Law, E.L.-C., Roto, V., Hassenzahl, M., Vermeeren, A.P.O.S., Kort, J.: Understanding, scoping and defining user experience: a survey approach. In: Proceedings of the 27th International Conference on Human Factors in Computing Systems - CHI 2009, p. 719. ACM Press, Boston (2009). https://doi.org/10.1145/1518701.1518813

16. Hassenzahl, M.: The hedonic/pragmatic model of user experience. In: Proceedings Towards a UX Manifesto, Lancaster, UK (2007)

17. Hassenzahl, M.: The thing and I: understanding the relationship between user and product. In: Blythe, M.A., Overbeeke, K., Monk, A.F., Wright, P.C. (eds.) Funology. HCIS, vol. 3, pp. 31-42. Springer, Dordrecht (2003). https://doi.org/10.1007/1-4020-2967-5_4

18. Huang, M., Ali, R., Liao, J.: The effect of user experience in online games on word of mouth: a pleasure-arousal-dominance (PAD) model perspective. Comput. Hum. Behav. 75, 329-338 (2017). https://doi.org/10.1016/j.chb.2017.05.015

19. Hsieh, J.-K., Hsieh, Y.-C., Chiu, H.-C., Yang, Y.-R.: Customer response to web site atmospherics: task-relevant cues, situational involvement and PAD. J. Interact. Mark. 28, 225-236 (2014). https://doi.org/10.1016/j.intmar.2014.03.001

20. Bruun, A., Ahm, S.: Mind the gap! Comparing retrospective and concurrent ratings of emotion in user experience evaluation. In: Abascal, J., Barbosa, S., Fetter, M., Gross, T., Palanque, P., Winckler, M. (eds.) INTERACT 2015. LNCS, vol. 9296, pp. 237-254. Springer, Cham (2015). https://doi.org/10.1007/978-3-319-22701-6_17

21. Kitchenham, B.: Procedures for performing systematic reviews, vol. 33 (2004)

22. Cyr, D., Head, M., Ivanov, A.: Design aesthetics leading to m-loyalty in mobile commerce. Inf. Manag. 43, 950-963 (2006). https://doi.org/10.1016/j.im.2006.08.009

23. Zhou, H., Fu, X.: Understanding, measuring, and designing user experience: the causal relationship between the aesthetic quality of products and user affect. In: Jacko, J.A. (ed.) HCI 2007. LNCS, vol. 4550, pp. 340-349. Springer, Heidelberg (2007). https://doi.org/10. 1007/978-3-540-73105-4_38

24. Bhandari, U., Chang, K., Neben, T.: Understanding the impact of perceived visual aesthetics on user evaluations: an emotional perspective. Inf. Manag. 56, 85-93 (2019). https://doi.org/ 10.1016/j.im.2018.07.003

25. Deng, P.: Affect in web interfaces: a study of the impacts of web page visual complexity and order. MIS Q. 34, 711 (2010). https://doi.org/10.2307/25750702

26. Frederick, D., Mohler, J., Vorvoreanu, M., Glotzbach, R.: The effects of parallax scrolling on user experience in web design. J. Usability Stud. 10, 9 (2015)

27. Wang, R., Sundar, S.S.: How does parallax scrolling influence user experience? A test of TIME (Theory of Interactive Media Effects). Int. J. Hum.-Comput. Interact. 34, 533-543 (2018). https://doi.org/10.1080/10447318.2017.1373457 
28. Lai, Y.-L., Kuan, K.K.Y., Hui, K.-L., Liu, N.: The effects of moving animation on recall, hedonic and utilitarian perceptions, and attitude. IEEE Trans. Eng. Manag. 56, 468-477 (2009). https://doi.org/10.1109/TEM.2009.2023454

29. Bonnardel, N., Piolat, A., Le Bigot, L.: The impact of colour on website appeal and users' cognitive processes. Displays 32, 69-80 (2011). https://doi.org/10.1016/j.displa.2010.12.002

30. Cyr, D., Head, M., Larios, H.: Colour appeal in website design within and across cultures: a multi-method evaluation. Int. J. Hum. Comput. Stud. 68, 1-21 (2010). https://doi.org/10. 1016/j.ijhcs.2009.08.005

31. Park, S.J., MacDonald, C.M., Khoo, M.: Do you care if a computer says sorry?: user experience design through affective messages, p. 10 (2012)

32. Fogg, B.J., Nass, C.: Silicon sycophants: the effects of computers that flatter. Int. J. Hum. Comput. Stud. 46, 551-561 (1997). https://doi.org/10.1006/ijhc.1996.0104

33. Mancini, M., et al.: Implementing and evaluating a laughing virtual character. ACM Trans. Internet Technol. 17, 1-22 (2017). https://doi.org/10.1145/2998571

34. Qiu, L., Benbasat, I.: Evaluating anthropomorphic product recommendation agents: a social relationship perspective to designing information systems. J. Manag. Inf. Syst. 25, 145-182 (2009). https://doi.org/10.2753/MIS0742-1222250405

35. Wagner, K., Nimmermann, F., Schramm-Klein, H.: Is it human? The role of anthropomorphism as a driver for the successful acceptance of digital voice assistants, p. 10 (2019)

36. Lee, S., Choi, J.: Enhancing user experience with conversational agent for movie recommendation: effects of self-disclosure and reciprocity. Int. J. Hum. Comput. Stud. 103, 95-105 (2017). https://doi.org/10.1016/j.ijhcs.2017.02.005

37. Dou, X., Sundar, S.S.: Power of the swipe: why mobile websites should add horizontal swiping to tapping, clicking, and scrolling interaction techniques. Int. J. Hum.-Comput. Interact. 32, 352-362 (2016). https://doi.org/10.1080/10447318.2016.1147902

38. Hassenzahl, M., Wiklund-Engblom, A., Bengs, A., Hägglund, S., Diefenbach, S.: Experience-oriented and product-oriented evaluation: psychological need fulfillment, positive affect, and product perception. Int. J. Hum.-Comput. Interact. 31, 530-544 (2015). https://doi.org/10.1080/10447318.2015.1064664

39. Hamari, J., Koivisto, J.: Why do people use gamification services? Int. J. Inf. Manag. 35, 419-431 (2015). https://doi.org/10.1016/j.ijinfomgt.2015.04.006

40. Suh, A., Wagner, C., Liu, L.: The effects of game dynamics on user engagement in gamified systems. In: 2015 48th Hawaii International Conference on System Sciences, pp. 672-681. IEEE, Waikoloa (2015). https://doi.org/10.1109/HICSS.2015.87

41. Anthropomorphism, n. (1885)

42. Sheldon, K.M., King, L.: Why positive psychology is necessary. Am. Psychol. 56, 216-217 (2001). https://doi.org/10.1037/0003-066X.56.3.216 